\title{
THREE-DIMENSIONAL COMPUTATIONAL PREDICTION OF VORTEX SEPARATION PHENOMENON INSIDE THE RANQUE-HILSCH VORTEX TUBE
}

\author{
Seyed Ehsan RAFIEE ${ }^{1}$, Mohammad Bagher Mohammad SADEGHIAZAD ${ }^{2}$ \\ Department of Mechanical Engineering, Urmia University of Technology, Urmia P.O. Box 57155-419, Iran \\ E-mails: ${ }^{1}$ s.e.rafiee@mee.uut.ac.ir (corresponding author), \\ seyed.ehsan.rafiee@gmail.com; ${ }^{2}$ dfimas@gmail.com
}

Received 10 December 2015; accepted 07 January 2016

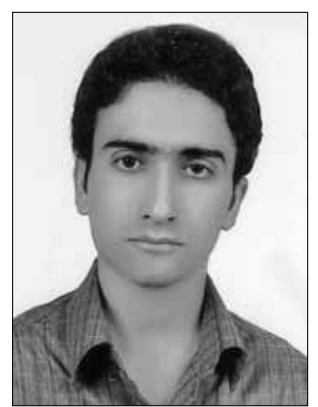

\section{Seyed Ehsan RAFIEE}

Date and place of birth: 1985, Kermanshah, Iran.

Education: B. S. degree in Mechanical Engineering from Razi University in 2010. M.S. and $P h D$ degrees in Mechanical Engineering (major in Energy Engineering), from Urmia University of Technology in 2012 and 2015, respectively. Affiliations and functions: Assistant Professor at Urmia University of Technology, in the Department of Mechanical Engineering. Research interests: fluid mechanics and thermodynamics.

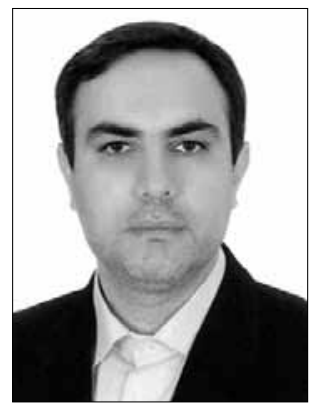

\author{
M. M. SADEGHIAZAD \\ Date and place of birth: 1976, Tabriz, Iran. \\ Education: B. S. degree in Mechanical Engineering from Tabriz University, in 1998. M. \\ S. and PhD degrees both in Mechanical Engineering (major in Fluid Mechanics), from \\ University of Tabriz in 2002 and 2007, respectively. \\ Affiliations and function: Associate professor in the Department of Mechanical Engineering \\ of Urmia University of Technology, Urmia, Iran. \\ Research interests: fluid mechanics, thermodynamics, artificial intelligence.
}

Abstract. The air separators are used to provide safe, clean and appropriate air to the helicopter's engine. In this operational study, the separation process inside a Ranque-Hilsch air separator cleaning system has been investigated to analyze the impact of choosing the appropriate turbulence model for predicting the separation process inside the air separator. This research is directed towards presenting a computational fluid dynamic explanation performed on a counter-flow air separator using air at different magnitudes of air flow fraction and applying different turbulence models. In a numerical investigation of counter-flow air separator, air has been chosen and its vortex separation phenomenon has been analyzed as a function of flow fraction. Furthermore, a numerical analysis to compare the outputs of a seven equation RSM turbulence model applied for the study of vortex separation of a counter-flow air separator with some two-equation turbulence methods, namely, k- $\varepsilon$ and k- $\omega$ model as well as LES has been presented. All of the turbulence numerical methods are seen to present and predict the same flow pattern inside an air separator, but, with various details. The results show that among the tested methods the RSM creates the most accurate separation pattern. The numerical results are validated by some available experimental data with good agreement.

Keywords: vortex tube, air separator, separation process, turbulence model, numerical simulation.

\section{Introduction}

The vortex tube is an uncomplicated device with no moving parts which is able to separate a pressurized operating fluid into two different streams, such as the dirty and the clean streams (or the hot and the cold). The separation process of the vortex tube air separator can be simplified as shown in Figure 1. 


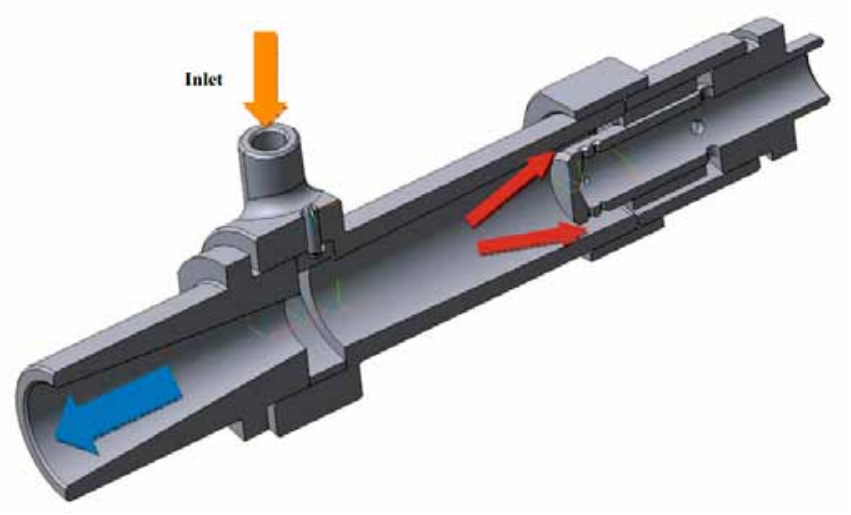

Fig. 1. A simplified plot of the vortex tube structure and the separation process

The separator system includes: a vortex chamber, some slots, a cold orifice, a working tube and a conical valve. There is a super rotational flow field with $10^{6} \mathrm{rpm}$ inside the air separator. First the fluid, which is compressed by a compressor, enters to the air separator via the nozzles (slots) and a powerful turbulent field is created during the tangential movement inside the main tube. The center of the vortex tube can be regarded as the axis of the rotation, and it can be said that when the pressurized gas layers are turning tangentially around this center, the operating gas will expand and the temperature will drop. During the rotational movement of the fluid layers, the separation process occurs and the pressurized gas is divided into two flows, namely the clean (cold) and the dirty (hot) flows. In the vortex tube cooling or cleaning systems, there is an orifice near the nozzles which brings out the cold flow from the cooling system. The rate of the clean or cold flow is controlled by a conical or throttle valve which is located at the hot side. The control valve controls the flow of the cold air through the warm air flow control.

An important parameter for this is:

$$
\alpha=\frac{\dot{m}_{c}}{\dot{m}_{i}},
$$

where $\alpha$ is the cold flow fraction. In Equation (1) the $\dot{m}_{c}$ and $\dot{m}_{i}$ are the cold flow rate and the rate of the inlet gas. The vortex tube air separator was invented by a French researcher, Ranque, in 1933. This device was geometrically improved by Rudolf Hilsch (1947). The details of the separation process inside the vortex tube air separator have not been clearly explained yet. In this article we utilize numerical models to explain the details of the separation process inside the air separator. Research on the vortex tube air separator has a long history; however, we will review a brief list of the most important works. The NIST real gas model (as a CFD model) was used to investigate the flow pattern inside the air separator by Dutta et al. (2011). The capabilities of different turbulence models $(k-\varepsilon$ and $S S T)$ for predicting the flow structures within the air separator were examined by Baghdad et al. (2011). Some variations in the temperature drops are seen when a bended main tube is used in the structure of the air separator. These variations are discussed in comparison with an air separator equipped with a straight main tube (Rafiee et al. 2015a; Bovand et al. 2014a and 2014b; Valipour et al. 2011). Skye et al. (2006) performed an experiment on the thermal and flow separation inside a commercial vortex tube. Three various classifications of the vortex tubes were adjusted as: (a) six cascade type RHVT; (b) vortex tube with threefold cascade situation; (c) conventional vortex tube, by Dincer (2011). Some factors regarding the vortex tube structure (the inlet of slots, the ratio of slots, the hot and cold exit areas, the rounding off edge radius, the internal radius of main tube and the convergent slots) were optimized by Rafiee and Sadeghiazad (2015), Rafiee et al. (2013), Pourmahmoud et al. (2012) and Im, Yu (2012). Suresh Kumar et al. (2014) optimized the hot outlet temperature for the straight air separator. Some refrigerant gases (R728, R32, R134a, R161, R744, R22, $\mathrm{NO}_{2}, \mathrm{CO}_{2}, \mathrm{O}_{2}, \mathrm{~N}_{2}$ and Air) have been examined in the vortex tube air separator and the thermal performance of the air separator has been studied and the best refrigerant gas has been determined by Han et al. (2013) and Pourmahmoud et al. (2013). This effect is studied in other research from different viewpoints using different gases (Thakare, Parekh 2015). MohammadI and Farhadi (2013) presented a laboratory study on the optimization of the nozzle diameter and the cold flow fraction. Rafiee and Sadeghiazad (2014a) managed some experimental setups to optimize the control valve structural parameters, such as the conical angle and the cone length, and proved that there are some optimized values which lead to the best thermal capability. Some research articles stated that the higher the number of the nozzles, the higher the thermal ability (Shamsoddini, Hossein Nezhad 2010). Chang et al. (2011) optimized the divergence angle of the main tube for a divergent air separator. The nozzle shape can be a strong and effective parameter which affects the cooling ability or the heating effectiveness of the air separator. The convergent nozzles (as a new shape) have been examined and optimized by Rafiee and Rahimi (2013). The impact of a new shape of vortex tube (double circuit vortex tube) has been numerically tested by Alekhin et al. (2015). Their results stated that there is an optimized angle for the convergent main tube producing the best cooling capacity. Xue et al. (2013a), and Rafiee and Sadeghiazad (2014b) proposed a new energy explanation to analyze the thermal distribution and the exergy density inside the air separator applying the measured flow factors along the hot tube. An optimization study has been done by Xue et al. (2013b) on the impact of the cold flow fraction which concentrates on the equal share of the rotating flows between 
the hot and the cold exhausts. The thermophysical parameters inside the vortex tube are comprehensively reported by Hamdy et al. (2015).

In the present work, we considered the advantage of utilizing the best turbulence model (for accurate prediction) and its clear role in the structural optimization of the air separator.

\section{Governing equations}

The extremely rotating and compressible flow field inside the air separator is considered as the main computational domain due to the creation of a three dimensional structure. A commercial code (Fluent 6.3.26) is employed to simulate the turbulent patterns inside the air separator using the RSM, standard $k-\varepsilon, k-\omega$ and LES turbulence models. Some turbulence models, such as the RSM and LES, are very accurate (in the rotational issues) but the CPU time is very high in these models. In addition, there is some research which indicates that the standard $k-\varepsilon$ is a relatively good turbulence model (regarding time and precision) and its results have an acceptable accuracy in order to establish a correct validation with experimental results (Rafiee et al. 2013). A numerical model has also been developed and is restricted by the following considerations: (a) the operating fluid properties are constant; (b) the flow field regime is assumed full turbulent; (c) the steady state condition is considered (with the exception of the LES simulation which is considered to be an unsteady process). With these conditions and assumptions, we can present the governing equations as the continuity (conservation of mass), momentum and energy equations, which can be expressed as:

$$
\begin{aligned}
& \frac{\partial}{\partial x_{j}}\left(\rho u_{j}\right)=0 ; \\
& \frac{\partial}{\partial x_{j}}\left(\rho u_{i} u_{j}\right)=-\frac{\partial p}{\partial x_{i}}+ \\
& \frac{\partial}{\partial x_{j}}\left[\mu\left(\frac{\partial u_{i}}{\partial x_{j}}+\frac{\partial u_{j}}{\partial x_{i}}-\frac{2}{3} \delta_{i j} \frac{\partial u_{k}}{\partial x_{k}}\right)\right] \\
& +\frac{\partial}{\partial x_{j}}\left(-\bar{\rho} \bar{u}_{i}^{\prime} \bar{u}_{j}^{\prime}\right) ; \\
& \frac{\partial}{\partial x_{i}}\left[u_{i} \rho\left(\frac{1}{2} u_{j} u_{j}+h\right)\right]=\frac{\partial}{\partial x_{j}}\left[u_{i}\left(\tau_{i j}\right)_{e f f}+k_{e f f} \frac{\partial T}{\partial x_{j}}\right] \\
& k_{e f f}=\frac{c_{p} \mu_{t}}{\operatorname{Pr}_{t}}+K .
\end{aligned}
$$

One of the assumptions needed to simplify heat transfer computations is the consideration of the operating fluid as an ideal gas, in which case there is the compressibility effect:

$$
p=\rho R T \text {. }
$$

Equations (6) and (7) below present detailed configurations of the dissipation rate $(\varepsilon)$ and the turbulence kinetic energy $(k)$ regarding the standard $k$ - $\varepsilon$ turbulence model.

$$
\begin{aligned}
& \frac{\partial}{\partial t}(\rho \varepsilon)+\frac{\partial}{\partial x_{i}}\left(\rho \varepsilon_{u_{i}}\right)=\frac{\partial}{\partial x_{j}}\left[\left(\mu+\frac{\mu_{t}}{\sigma_{\varepsilon}}\right) \frac{\partial \varepsilon}{\partial x_{j}}\right]+ \\
& C_{1 \varepsilon} \frac{\varepsilon}{k}\left(G_{k}+C_{3 \varepsilon} G_{b}\right)-C_{2 \varepsilon} \rho \frac{\varepsilon^{2}}{k} \\
& \frac{\partial}{\partial t}(\rho k)+\frac{\partial}{\partial x_{i}}\left(\rho k u_{i}\right)=\frac{\partial}{\partial x_{j}}\left[\left(\mu+\frac{\mu_{t}}{\sigma_{k}}\right) \frac{\partial k}{\partial x_{j}}\right] \\
& +G_{k}+G_{b}-\rho \varepsilon-Y_{M}
\end{aligned}
$$

Here $\mathrm{Gk}$ and $\mathrm{Gb}$ stand for the turbulence kinetic energy generation regarding the gradients of the mean velocity and the buoyancy effect, respectively. Also, YM is the fluctuating stretch contribution in compressible turbulence to the overall rate of dissipation. Additionally, there are two constants $-\mathrm{C} 1 \varepsilon$ and $\mathrm{C} 2 \varepsilon$. It should be said that $\sigma \varepsilon$ and $\sigma \mathrm{k}$ are the Prandtl numbers (turbulent). Now, we need to define the turbulent viscosity $\mu \mathrm{t}$, so:

$$
\mu_{t}=\rho C_{\mu} \frac{k^{2}}{\varepsilon} \text {. }
$$

In this equation, $C_{\mu}=0.09$ and is a constant value. Also, the constants in Equation (6) and (7) are as follows: $\sigma_{\varepsilon}=1.3, C_{1 \varepsilon}=1.44, C_{2 \varepsilon}=1.92, C_{\mu}=0.09, \sigma_{k}=1.0, \sigma_{\varepsilon}=1.3$.

The main transport equations of the Reynolds stresses (for RSM), $\rho \overline{u_{i}^{\prime} u_{j}^{\prime}}$, can be written as:

$$
\begin{aligned}
& \frac{\partial}{\partial t}\left(\rho \overline{u_{i}^{\prime} u_{j}^{\prime}}\right)+\underbrace{\frac{\partial}{\partial x_{k}}\left(\rho u_{k} \overline{u_{i}^{\prime} u_{j}^{\prime}}\right.}_{C_{i j}})= \\
& \underbrace{-\frac{\partial}{\partial x_{k}}\left[\rho \overline{u_{i}^{\prime} u_{j}^{\prime} u_{k}^{\prime}}+p\left(\delta_{k j} u_{i}^{\prime}+\delta_{i k} u_{j}^{\prime}\right)\right]}_{D_{T, i j}}+\underbrace{\left.\frac{\partial}{\partial x_{k}}\left[\mu \frac{\partial}{\partial x_{k}} u_{i}^{\prime} u_{j}^{\prime}\right)\right]}_{D_{L, i j}} \\
& \underbrace{-\rho\left(\overline{u_{i}^{\prime} u_{k}^{\prime}} \frac{\partial u_{j}}{\partial x_{k}}+\overline{u_{j}^{\prime} u_{k}^{\prime}} \frac{\partial u_{i}}{\partial x_{k}}\right)}_{P_{i j}}-\underbrace{\rho \beta\left(g_{i} \overline{u_{j}^{\prime} \theta}+g_{j} \overline{u_{i}^{\prime} \theta}\right)}_{G_{i j}}+ \\
& \underbrace{p\left(\frac{\partial u_{i}^{\prime}}{\partial x_{j}}+\frac{\partial u_{j}^{\prime}}{\partial x_{i}}\right)}_{\phi_{i j}} \\
& -2 \mu \underbrace{\overline{\frac{\partial u_{i}^{\prime}}{\partial x_{k}} \frac{\partial u_{j}^{\prime}}{\partial x_{k}}}}_{\varepsilon_{i j}}-\underbrace{2 \rho \Omega_{k}\left(u_{j}^{\prime} u_{m}^{\prime} \varepsilon_{i k m}+u_{i}^{\prime} u_{m}^{\prime} \varepsilon_{j k m}\right)}_{F_{i j}}+S_{u s e r} .
\end{aligned}
$$

Where $S_{u s e r}, F_{i j}, \varepsilon_{i j}, \phi_{i j}, G_{i j}, P_{i j}, D_{L, i j}, D_{T, i j}, C_{i j}$ and $\frac{\partial}{\partial t}\left(\rho \overline{u_{i}^{\prime} u_{j}^{\prime}}\right)$ are considered as User-Defined Source Term, Production By System Rotation, Dissipation, Pressure Strain, Buoyancy Production, Stress Production, Molecular Diffusion, Turbulent Diffusion, Convection and Local Time Derivative, respectively. 


\section{Physical model description}

\section{1. 3D CFD model}

The three-dimensional model of the air separator is created and developed based on the experimental air separator cooling system used by Skye et al. (2006). The model of the vortex tube air separator used in their tests was ExairTM 708 slpm. Figure 2 depicts a schematic representation of the real model of the air separator used by Skye et al. (2006). The structural dimensions of the air separator are summarized in Table 1. Figure 3a shows the created computational grids. As seen in Figure 3a, the created CFD model of the air separator uses the structured grid pattern to divide the domain into cubic units (with the exception of the volume units around the center line which are triangular prism units); because of this fact, the calculations of a domain with an unstructured mesh grid system usually take more CPU time than the calculation of the domain with the structured mesh grid arrangement. Also, the structured arrangements are more accurate compared to the unstructured ones.

Table 1. Geometrical details of the CFD model which are similar to the experimental dimensions

\begin{tabular}{ll}
\hline Area of hot exit & $95 \mathrm{~mm}^{2}$ \\
\hline Diameter of hot exit & $11 \mathrm{~mm}$ \\
\hline Diameter of cold exhaust & $6.2 \mathrm{~mm}$ \\
\hline Slot area & $8.2 \mathrm{~mm}^{2}$ \\
\hline Width of nozzle & $1.41 \mathrm{~mm}$ \\
\hline Area of cold exit & $30.3 \mathrm{~mm}^{2}$ \\
\hline Height of nozzle & $0.97 \mathrm{~mm}$ \\
\hline Diameter of main tube & $11.4 \mathrm{~mm}$ \\
\hline Length of main tube & $106 \mathrm{~mm}$ \\
\hline
\end{tabular}

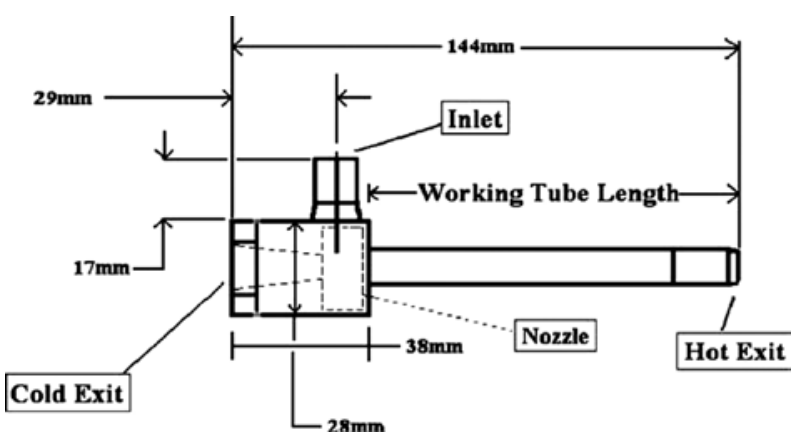

Fig. 2. Structured diagram of the air separator used in the tests

In the computations, the velocity pressure coupling uses the SIMPLE algorithm. Also, the convective items have been discretized using the second order upwind scheme. The minimum value for the convergence standard regarding the turbulence and the velocity quantities and the continuity equation was $10^{-6}$ and $10^{-13}$ for the energy terms. One of the advantages of the created CFD a)

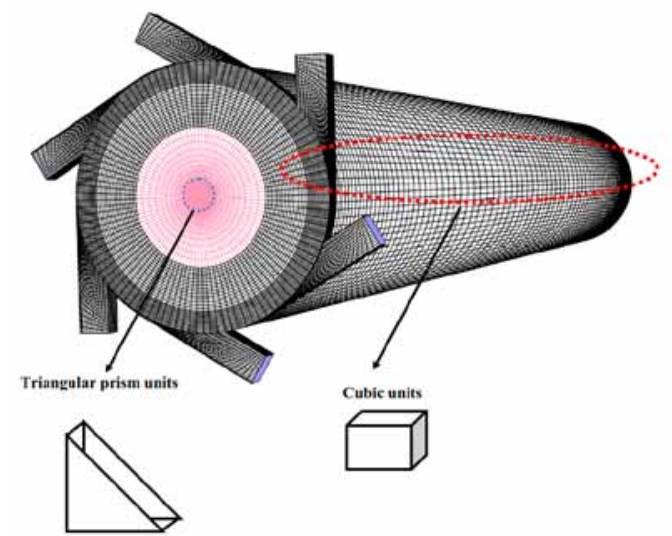

b)

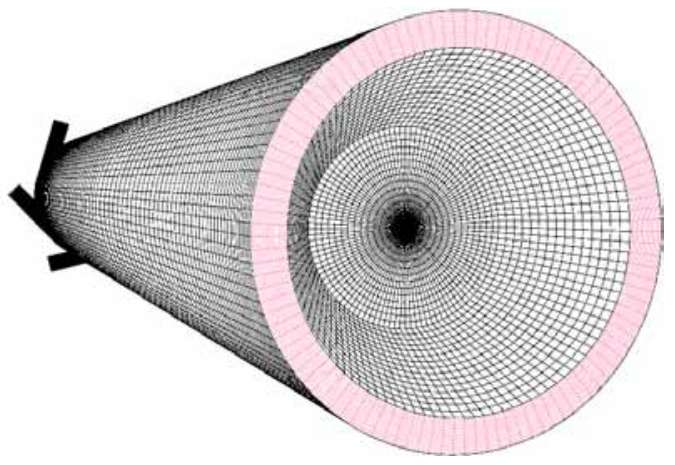

c)

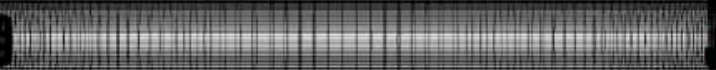

Fig. 3. a) Mesh arrangement of the CFD model, b) end view of the CFD model (hot control valve side), c) longitudinal arrangement of the mesh grids

model is that the hot control valve is completely modeled (as seen in Fig. 3b) which leads to a realistic modeling of the flow pattern inside the air separator (as much as possible). Because of the complexity of the flow field inside the air separator, some special combinations of the relaxation factors are used to simulate the separation process inside the device. The ranges of the relaxation factors are as follows: pressure ( 0.1 to 0.2$)$, density ( 0.1 to 1$)$, body force (0.1 to 1$)$, momentum ( 0.1 to 0.6 ) and energy ( 0.1 to 1 ). In this CFD model, the mesh grids are finer (Fig. 3c, for more accuracy), close to the cold and the hot exhausts where the cold and hot temperatures are measured.

\section{2. Grid independence study, boundary conditions and validation (using the RSM)}

A careful analysis for the grid-independence process of the CFD computations is performed to concentrate on the validity and the accuracy of the CFD outputs. During the grid-independence test, 3D CFD models are created based on various average unit cell volumes. For this reason, four grid systems are created and analyzed to consider the most accurate grid numbers for the CFD models. The thermal and velocity investigations have 
been done for four different average unit cell volumes. In this case, the optimum cold flow fraction is 0.3 since the highest cooling power can be found at this cold flow fraction. Therefore, the independence study is conducted at this cold flow fraction $(\alpha=0.3)$. The variations of the two main parameters, namely: the maximum tangential velocity and the cold exit temperature difference, are considered for different unit cell volumes, as shown in Figure 4 and Figure 5. As seen in Figure 4 and Figure 5, the application of the numerical models with the average unit cell volumes smaller than $0.0257 \mathrm{~mm}^{3}$ (which corresponds to 287000 cells) cannot make a big difference in the results. With this average cell volume $\left(0.0257 \mathrm{~mm}^{3}\right)$, the difference between the results is small, and the 287000 cells can present both accuracy and efficiency, so it average has been applied for all the models in this research.

Figure 6 shows a schematic diagram of the boundary conditions used in the vortex tube air separator CFD model. The mass-flow-inlet boundary condition is considered for the injectors with the mass flow rate of 8.35 gr. $^{-1}$ (4.8 bar) and the temperature at the slots adjusted at the ambient temperature of $294.2 \mathrm{~K}$ (based on experimental data). The no-slip situation is applied on the air separator's walls. There are two types of boundary

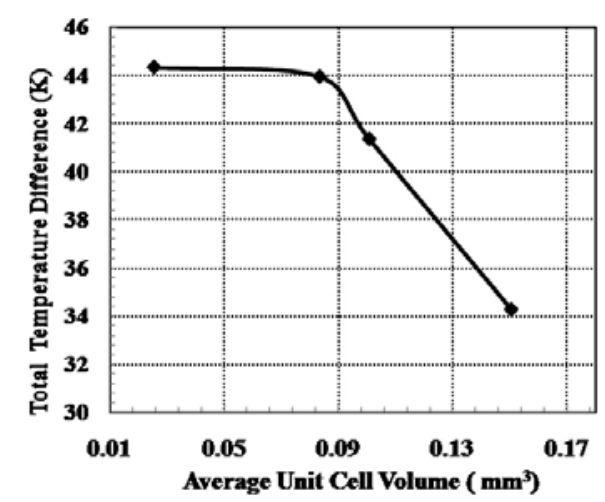

Fig. 4. Grid independence analysis of total temperature difference (using the RSM) conditions which can be used at the slot surfaces, namely: the Pressure-Far-Field and the Pressure-Outlet. When the pressure values at the cold and hot exhausts are measured in the experiments, the scientists can use the Pressure-Outlet boundary condition to perform the computations. This method can be applied by those who have access to laboratory data.

In this paper, we introduce a boundary condition method that can be used by researchers who do not have access to laboratory data, in other words, this article introduces a simple way to analyze a real model of the air separator without even knowing the pressure values at the exhausts. There is no need for the pressure values when the model is created based on the pressure-farfield boundary conditions. Our aim is to prove that the results of the two methods (Pressure-Far-Field and Pressure-Outlet) are consistent with each other.

In the CFD model of the vortex tube air separator, the cold flow fraction should be changed to achieve different efficiency levels whether the Pressure-Far-Field or the Pressure-Outlet is used. The procedure to change the cold flow fraction during the application of different boundary conditions is as follows:

a) Pressure-Outlet: achieved by considering a fixed pressure value at the cold outlet and changing

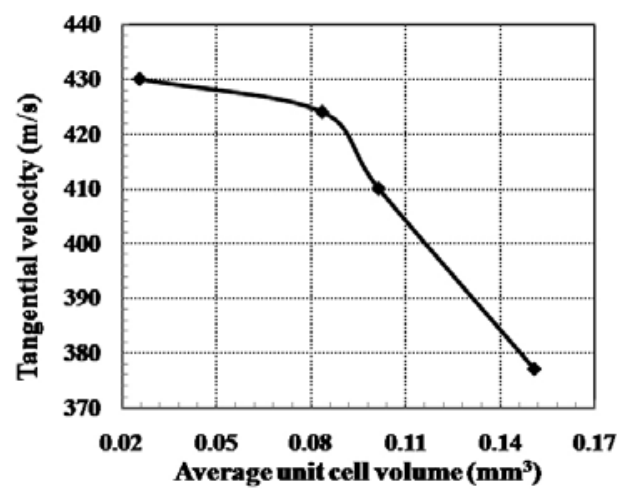

Fig. 5. Grid independence analysis of maximum swirl velocity (using the RSM)

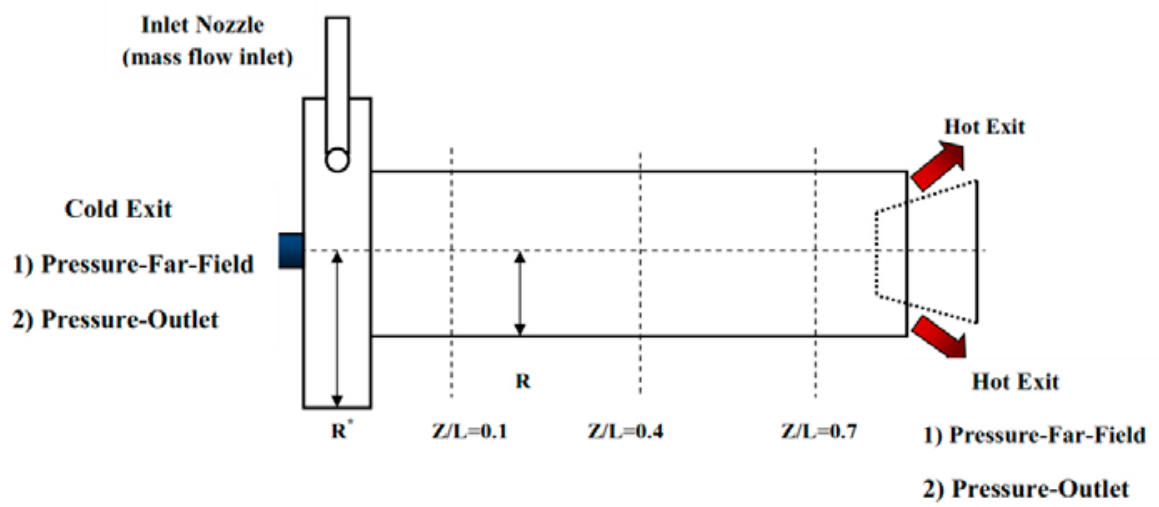

Fig. 6. Details of the boundary conditions used in the computations 
the pressure at the hot outlet (according to experimental values);

b) Pressure-Far-Field: obtained by adjusting a constant cold exit area and varying the hot area;

c) Experimental model: achieved by the control valve performance.

So, it can be seen that the second mode (the Pressure-Far-Field) is the option closest to the experimental process. In order to compare the mentioned boundary conditions, a comparison between the numerical outputs (cold and hot temperatures resulting from both boundary conditions) and experimental results (according to Skye et al. 2006) are shown in Figure 7 and Figure 8. All structural factors (as seen in Table 1) are completely similar for real and the CFD models. The only difference between the CFD models is the type of the boundary conditions, the operating conditions are adjusted as the real conditions. The adiabatic boundary condition is applied to the main tube wall (the outer surface) and the convection heat transfer between the air separator and the ambient is neglected, which is the main difference between the computational and real models. As depicted in Figure 7 and Figure 8, the

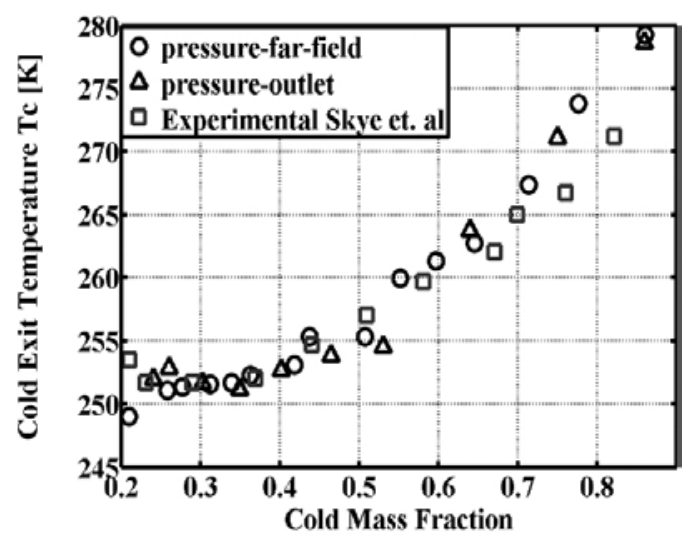

Fig. 7. Experimental cold exit temperature compared with the numerical values of the $3 \mathrm{D}$ CFD models applying both types of boundary conditions

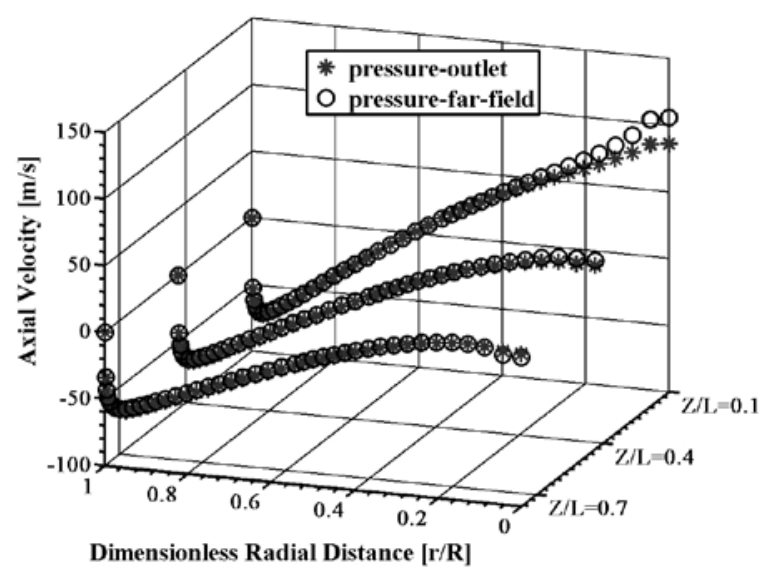

Fig. 9. Comparison of the axial velocities at different longitudinal sections regarding two different boundary conditions results of the $3 \mathrm{D}$ computations (for both boundary conditions) are in agreement with the experimental outputs and all the predicted values are within $7 \%$ of the laboratory results, which is within the acceptable range. Figure 7 and Figure 8 show that the developed model is an accurate model that produces reliable results using both boundary conditions (the Pressure-FarField or the Pressure-Outlet is used).

In order to present a complete comparison between the Pressure-Far-Field and the Pressure-Outlet boundary conditions, some parameters such as axial velocity, tangential velocity, total pressure and total temperature at three different longitudinal sections $(\mathrm{z} / \mathrm{L}=0.1,0.4$ and 0.7 as in Fig. 6) of the working tube have been studied as a function of $\mathrm{r} / \mathrm{R}$ (dimensionless radial distance), as well as the total temperature on the wall of the vortex tube air separator. The axial and rotational velocities, the total pressure and temperature distributions in different axial sections are shown in Figures 9-12. These figures illustrate a comparative presentation of the CFD results obtained by employing two different boundary conditions and one can see the good adjustability of the results for both models.

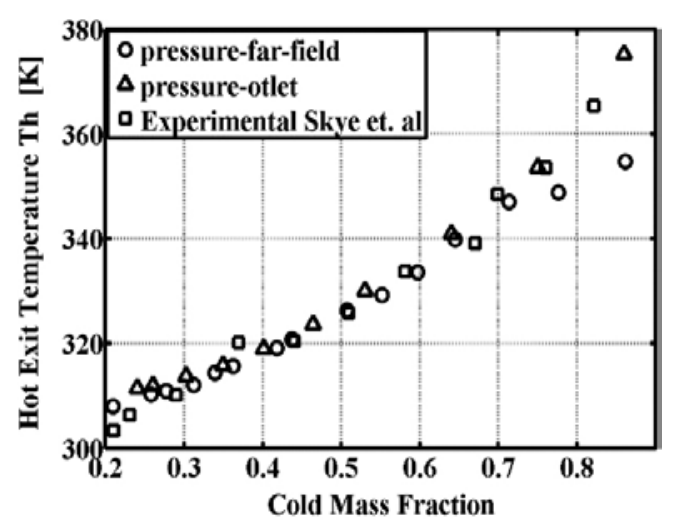

Fig. 8. Experimental hot exit temperature compared with the numerical values of the $3 \mathrm{D}$ CFD models applying both types of boundary conditions

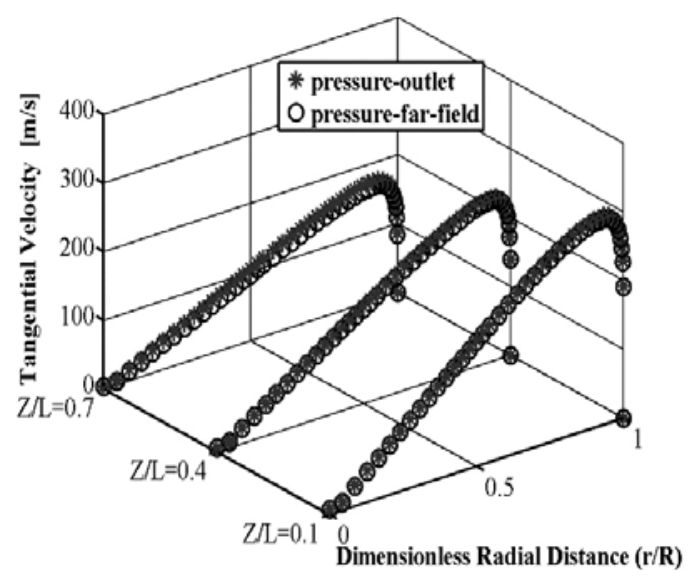

Fig. 10. Comparison of the tangential velocities at different longitudinal sections regarding two different boundary conditions 


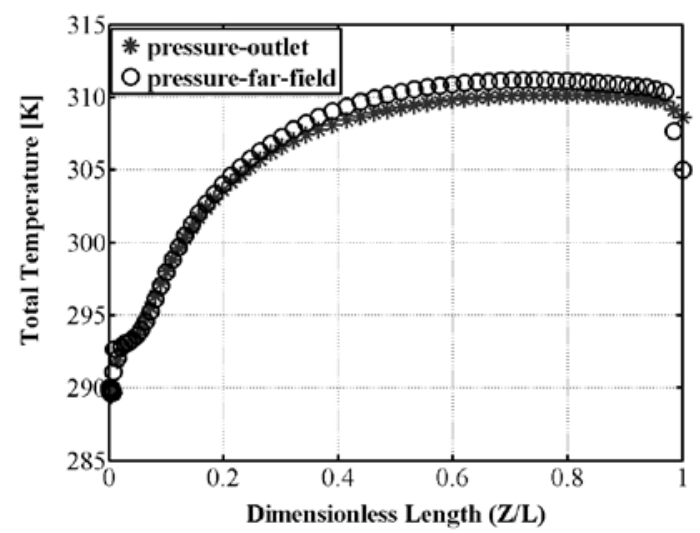

Fig. 11. Comparison of the total pressures at different longitudinal sections regarding two different boundary conditions

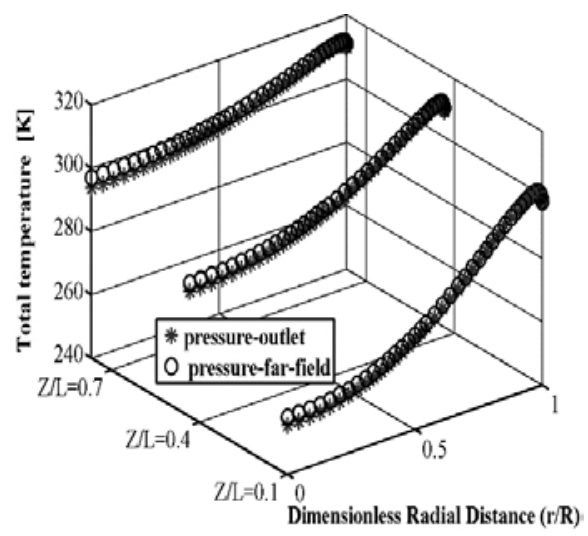

Fig. 12. Comparison of the total temperatures at different longitudinal sections regarding two different boundary conditions

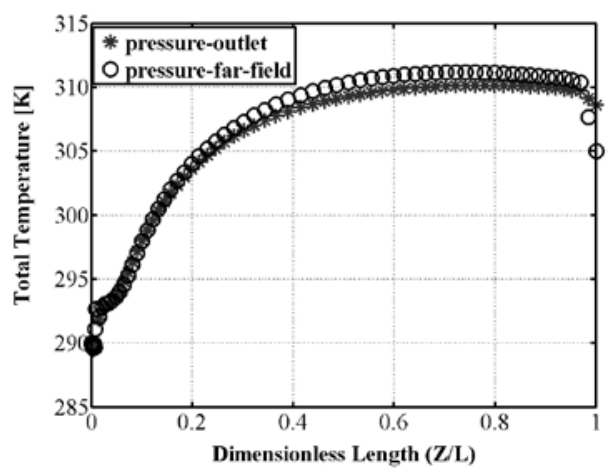

Fig. 13. Indication of the total temperature on the wall of the main tube for two different boundary conditions

Figure 13 indicates the variation of the total temperature on the wall of the tube for both boundary conditions compared (Pressure-Far-Field and Pressure-Outlet). In this case there is also good agreement between both CFD models.

Figures 9-13 prove that both types of boundary conditions have almost the same results and either of them can be used during the numerical process. The Pressure-Far-Field boundary condition however is more usable than the other one because, in most cases, there is no access to the pressure values of the exhausts (this method does not need the outlet pressure). So in the rest of this study, the Pressure-Far-Field boundary condition is applied for all numerical computations.

\section{Results (effect of different turbulence models)}

In the present study, the air separator thermal performance in terms of cold and hot exhaust temperatures $\left(T_{c}\right.$ and $T_{h}$ ) is analyzed, also the cold and hot power separation rates $\left(\dot{Q}_{c}\right.$ and $\left.\dot{Q}_{h}\right)$ are predicted numerically. This prediction is based on different operating turbulence models which are used as the operating methods. The main objective of this investigation is to achieve the most accurate prediction of cooling and heating capacities by changing the type of the operating turbulence model which controls the computation process of the energy separation rate inside the commercial vortex tube. Figure 14 presents the variation of the cold temperature difference $\Delta T_{c}\left(T_{i}-T_{c}\right)$ at the exhaust of the cold orifice as a function of the cold flow fraction. As seen in Figure 14, the trend of the experimental curve shows that the temperature difference $\Delta \mathrm{T}_{\mathrm{c}}$ of the cold exit decreases with the increasing cold flow fraction (for the cold flow fraction values greater than 0.36 ). This means that cold temperature $T_{c}$ increases for the cold flow fraction values greater than 0.36 . In the case of the RSM, there is a favorable agreement between the experimental results and the CFD outputs which proves that the RSM model is an accurate and reliable model to predict the thermal performance of the vortex tube air separator. The previous $3 \mathrm{D}$ model is used to analyze and predict the energy and gas separations inside the air separator using other operating turbulence models including $k-\varepsilon$, $k-\omega$ and LES. As the results of Figure 14 illustrate, all methods predict a lower cold temperature difference $\Delta \mathrm{T}_{\mathrm{c}}$ than the experimental results. Therefore, the use of these operating turbulence models instead of the RSM leads to a lower accuracy, for example, the cold exhaust temperature difference for the RSM as the operating turbulence model is $41.94 \mathrm{~K}$, in this way if LES or $k-\varepsilon$ is applied to predict the flow separation of the air separator, the cold temperature difference decreases to around 3 or $9 \mathrm{~K}$, respectively (for $\alpha=0.37$ ). Figure 14 indicates that the minimum possible difference between the experimental results and numerical predictions (cold temperature difference) corresponds to the RSM turbulence model.

This prediction emphasizes that when the vortex tube air separator is utilized as a refrigerator system with air, the RSM turbulence model is the best choice among the methods mentioned because it produces the most accurate cold temperature in comparison with the other methods. The minimum value of the cold exit temperature (or the maximum cold temperature difference) for 


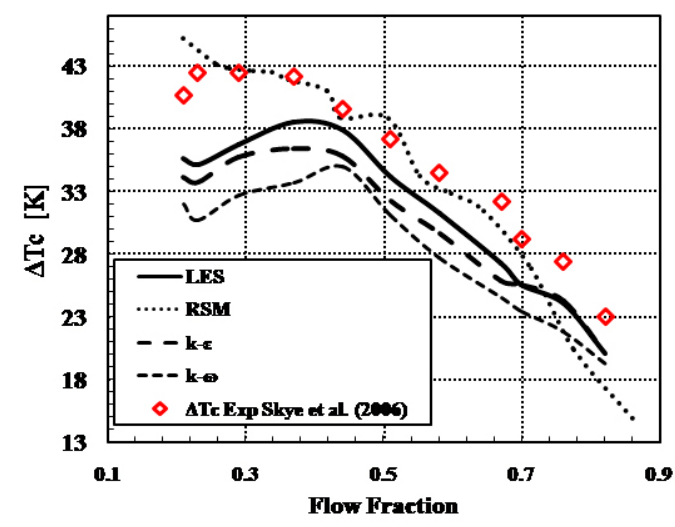

Fig. 14. Variation of the cold temperature difference for different types of operating turbulence models as a function of the cold flow fraction (compared with experimental results)

all methods is located in the cold flow fraction range of $0.29-0.37$. Figure 15 presents the behavior of the temperature curves regarding the hot gas which escapes from the hot exhaust (as a function of the cold mass fraction) when applying different turbulence models.

As shown in Figure 15, all of the turbulence models present the same general tendency at the hot exit at different cold flow fractions. Figure 15 shows that the hot exit temperature difference $\Delta \mathrm{T}_{\mathrm{h}}\left(\mathrm{T}_{\mathrm{h}}-\mathrm{T}_{\mathrm{i}}\right)$ enhances with increasing cold flow fraction for all methods. Figure 15 indicates that if the vortex tube air separator is used as the heating system, there is no difference between the turbulence models mentioned (excluding the RSM). Therefore, a turbulence model which has a lower cost than other models should be chosen, because there is no difference between the methods from the perspective of heating usage. In the case of the RSM, the experimental and numerical hot temperature differences are in perfect agreement, so we can trust the numerical method to predict the hot temperatures as well as the cold exit temperature. The temperature range of the hot outlet for different methods $(k-\varepsilon, k-\omega$ and LES) that exit from the hot exhaust is found to be between $300 \mathrm{~K}$ and $378.86 \mathrm{~K}$ (the hot temperature difference is found to be between 5.87 and 84.68). Table 2 summarizes the numerical results of

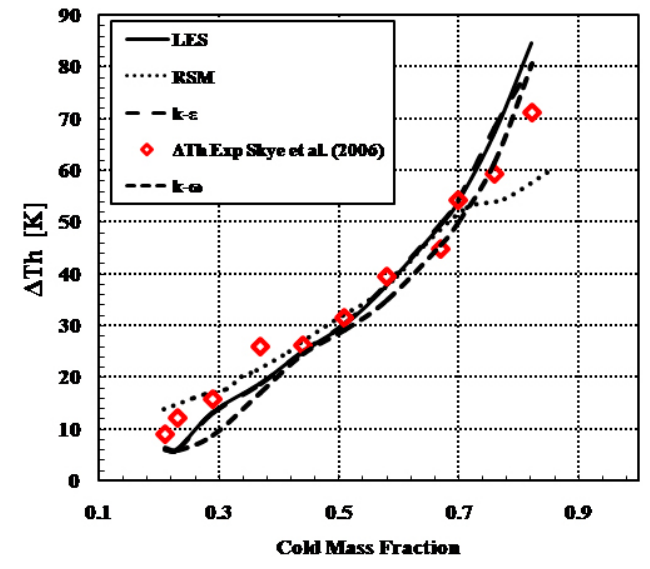

Fig. 15. Variation of the hot exhaust temperature difference for different types of operating turbulence models as a function of cold flow fraction (compared with the experimental results)

the hot and cold exhaust temperatures $\left(T_{c}\right.$ and $\left.T_{h}\right)$ and their differences $\left(\Delta T_{c}\right.$ and $\left.\Delta T_{h}\right)$ for all types of operating turbulence models at the cold flow fraction of $\alpha=0.3$. The results show that the RSM turbulence model shows the maximum accuracy, results to closest to the experimental data.

Table 3 offers a practical suggestion for the type determination of the operating turbulence model to achieve the maximum accuracy for cooling or heating calculations. In Table 3, the turbulence models which have a higher accuracy for heating $\left(\Delta T_{h}\right)$ and cooling $\left(\Delta T_{c}\right)$ calculations are arranged from top to bottom. However, from the time standpoint, it seems that $k-\varepsilon$ is more appropriate and affordable for the use in the procedure of calculations but other operating methods have a higher accuracy and reliability despite the higher CPU times.

Table 3. The accuracy of the turbulence models regarding computations for cooling and heating capabilities

\begin{tabular}{ll}
\hline Cooling preference & Heating preference (relative) \\
\hline RSM & RSM \\
\hline LES & LES \\
\hline $\mathrm{k}-\varepsilon$ & $\mathrm{k}-\varepsilon$ \\
\hline $\mathrm{k}-\omega$ & $\mathrm{k}-\omega$ \\
\hline
\end{tabular}

Table 2. Numerical temperature separations regarding different types of the operating turbulence models with the cold mass fraction $\alpha=0.3$

\begin{tabular}{llllll}
\hline Type of Gas & \multicolumn{1}{c}{$\begin{array}{c}\text { Average } \\
\text { Cold Exhaust } \\
\text { Temperature (K) }\end{array}$} & $\begin{array}{c}\text { Average } \\
\text { Hot Exhaust } \\
\text { Temperature (K) }\end{array}$ & $\Delta T_{c}(\mathrm{~K})$ & $\Delta T_{h}(\mathrm{~K})$ & $\Delta T_{t}(\mathrm{~K})$ \\
\hline Experimental Air & 251.7 & 310.14 & 42.5 & 15.94 & 58.44 \\
\hline RSM & 251.33 & 310.95 & 42.87 & 16.75 & 59.62 \\
\hline LES & 257.48 & 307.5 & 36.72 & 13.3 & 50.02 \\
\hline k- $\varepsilon$ & 258.45 & 307.28 & 35.75 & 13.08 & 48.83 \\
\hline k- $\omega$ & 261.5 & 302.97 & 32.7 & 8.77 & 41.47 \\
\hline
\end{tabular}




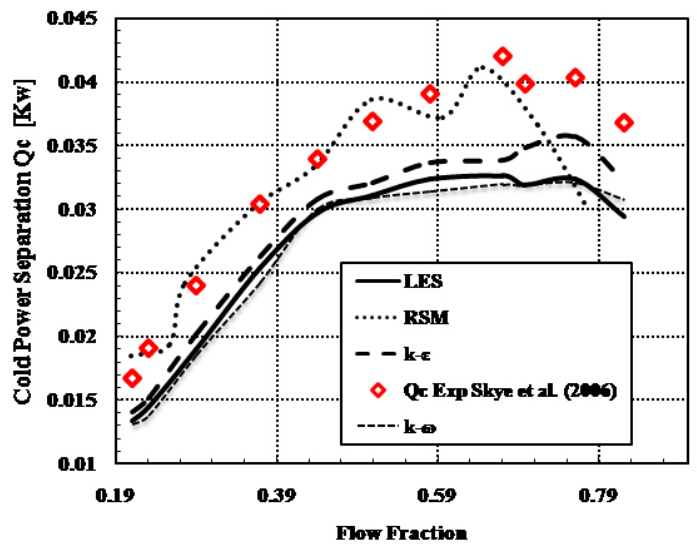

Fig. 16. Variation of the cold power separation for different types of operating turbulence models as a function of cold flow fraction (compared with the experimental results)

Another parameter that illustrates air separator performance is the energy separation rate at the hot and cold exits $\left(\dot{Q}_{c}\right.$ and $\left.\dot{Q}_{h}\right)$, which can be evaluated as follows:

$$
\begin{aligned}
& \dot{Q}_{c}=\dot{m}_{c} c_{p}\left(T_{i}-T_{c}\right) ; \\
& \dot{Q}_{h}=\dot{m}_{h} c_{p}\left(T_{h}-T_{i}\right),
\end{aligned}
$$

here, $c_{p}$ is the gas specific heat.

Figures 16 and 17 depict the cold and hot power separation $\left(\dot{Q}_{c}\right.$ and $\left.\dot{Q}_{h}\right)$ variations of the vortex tube air separator when applying different operating turbulence models (in comparison with experimental values). Both the experimental results and the CFD models show that the maximum power separation occurs at the cold fraction of about 0.65 . The rate of energy separation enhances with the increasing cold flow fraction in the range of $0.21-0.65$. For the cold flow fraction values greater than 0.7 , the increasing trends of the power separation stop and the thermal rates decrease with the increase in the cold flow fraction. The resulting total temperature contours are plotted as seen in Figure 18 for the RSM simulation. This Figure shows the cold core and the hot peripheral flow along the main tube of the vortex tube air separator. The following assumptions are made: the inlet mass flow rate is $8.34 \mathrm{~g} \mathrm{~s}^{-1}$, the inlet temperature is $294.2 \mathrm{~K}$ and the working fluid is air. The minimum and maximum total temperatures produced under these operating conditions are $250.24 \mathrm{~K}$ and $311.5 \mathrm{~K}$, respectively. The total temperature contour plotted in Figure 18 is related to $\alpha=0.3$ (which is the optimum cold flow fraction); this means that the machine produces the maximum cooling capacity in this cold flow fraction.

\section{Conclusion}

In this study, a 3D CFD model was improved to investigate the thermal and gas separations inside the vortex tube air separator (as a predictive tool). This is a developed

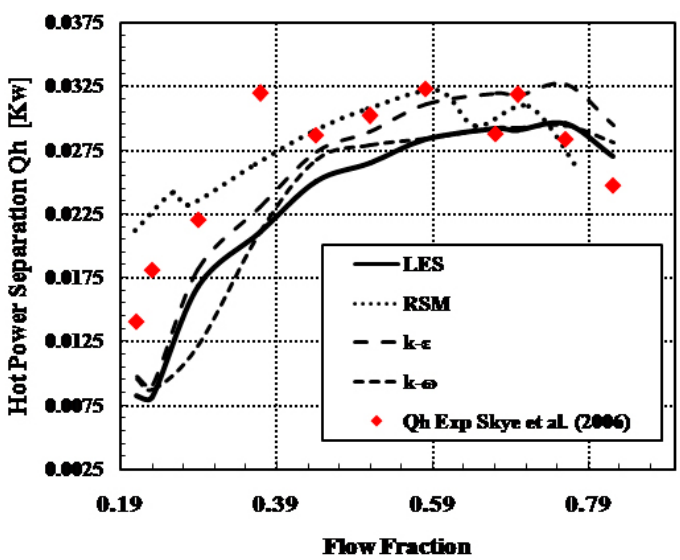

Fig. 17. Variation of the hot power separation for different types of operating gases as a function of the cold flow fraction (compared with experimental results)
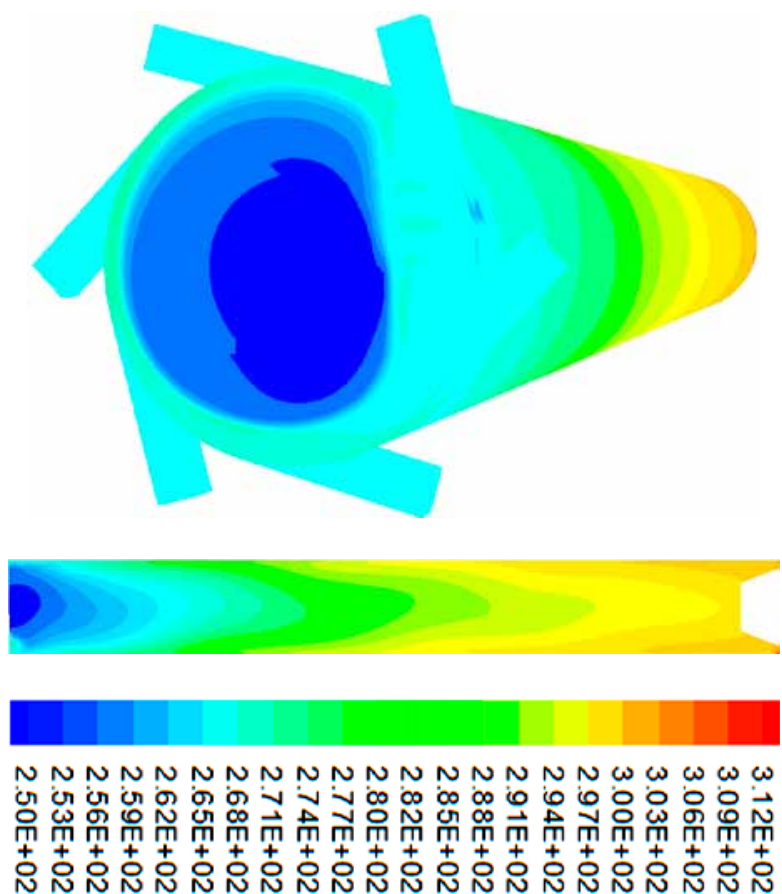

Fig. 18. Temperature distribution inside the vortex tube operating with compressed air, $\alpha=0.3$, using the RSM simulation

3D numerical model restricted by the following considerations: (a) the operating fluid properties are constant; (b) the flow field regime is assumed to be full turbulent; (c) the steady state condition is considered (with the exception of the LES simulation which is considered as an unsteady process). A commercial code (Fluent 6.3.26) is employed to simulate the turbulent patterns inside the air separator using different turbulence models, namely: $\mathrm{k}-\varepsilon$ and $\mathrm{k}-\omega$, LES (Large Eddy Simulation) and RSM (Reynolds Stress Model). The study on the effects of using different turbulence models (as the operating condition) on the cooling and heating computations of an air separator was the main objective of this research. The results of this study can help researchers choose the best 
type of the operating turbulence model (as a predictive tool) for air separator computations to achieve the highest accuracy and reliability. The conclusions are that for the computations regarding the cooling or heating the special zone by means of the vortex tube air separator, the RSM is the best choice and provides the maximum accuracy for a correct estimation. In this study, a comprehensive comparison between two different types of boundary conditions for the hot and the cold exhausts, i.e. the Pressure-Far-Field and Pressure-Outlet, was performed. When the pressure values at the cold and hot exhausts are measured in experiments, the scientists can use the Pressure-Outlet boundary condition to perform the computations. This method is applicable for those who have access to laboratory data. In this paper, we introduce a boundary condition method that can be used by researchers who do not have access to laboratory data, in other words, this article introduces a simple way to analyze a real model of the air separator without even knowing the pressure values at the exhausts. There is no need for the pressure values when the model is created based on the pressure-far-field boundary condition. The comparison of obtained numerical results and the available measured experimental data revealed a good and reliable agreement.

\section{References}

Alekhin, V.; Bianco, V.; Khait, A.; Noskov, A. 2015. Numerical investigation of a double-circuit Ranque-Hilsch vortex tube, International Journal of Thermal Sciences 89: 272-282. http://dx.doi.org/10.1016/j.ijthermalsci.2014.11.012

Baghdad, M.; Ouadha, A.; Imine O., et al. 2011. Numerical study of energy separation in a vortex tube with different RANS models, Thermal Sciences 50(12): 2377-2385. http://dx.doi.org/10.1016/j.ijthermalsci.2011.07.011

Bovand, M.; Valipour, M. S.; Dincer, K., et al. 2014a. Numerical analysis of the curvature effects on Ranque-Hilsch vortex tube refrigerators, Applied Thermal Engineering 65(1-2): 176-183. http://dx.doi.org/10.1016/j.applthermaleng.2013.11.045

Bovand, M.; Valipour, M. S.; Eiamsa-ard, S., et al. $2014 \mathrm{~b}$. Numerical analysis for curved vortex tube optimization, International Communications in Heat and Mass Transfer 50: 98-107. http://dx.doi.org/10.1016/j.icheatmasstransfer.2013.11.012

Chang, K.; Li, Q.; Zhou, G.; Li, Q., et al. 2011. Experimental investigation of vortex tube refrigerator with a divergent hot tube, Refrigeration 34(1): 322-327. http://dx.doi.org/10.1016/j.ijrefrig.2010.09.001

Dincer, K. 2011. Experimental investigation of the effects of threefold type Ranque-Hilsch vortex tube and six cascade type Ranque-Hilsch vortex tube on the performance of counter flow Ranque-Hilsch vortex tubes, Refrigeration 34(6): 1366-1371. http://dx.doi.org/10.1016/j.ijrefrig.2011.05.008

Dutta, T.; Sinhamahapatra, K. P.; Bandyopadhyay, S. S. 2011. Numerical investigation of gas species and energy separation in the Ranque-Hilsch vortex tube using real gas model, Refrigeration 26(8): 2118-2128.

http://dx.doi.org/10.1016/j.ijrefrig.2011.06.004
Han, X.; Li, N.; Wu, K., et al. 2013. The influence of working gas characteristics on energy separation of vortex tube, $A p$ plied Thermal Engineering 61(2): 171-177. http://dx.doi.org/10.1016/j.applthermaleng.2013.07.027

Hilsch, R. 1947. The use of expansion of gases in a centrifugal field as a cooling process, Review of Scientific Instruments 18: 108-113. http://dx.doi.org/10.1063/1.1740893

Im, S. Y.; Yu, S. S. 2012. Effects of geometric parameters on the separated air flow temperature of a vortex tube for design optimization, Energy 37(1): 154-160. http://dx.doi.org/10.1016/j.energy.2011.09.008

Mohammadi, S.; Farhadi, F. 2013. Experimental analysis of a Ranque-Hilsch vortex tube for optimizing nozzle numbers and diameter, Applied Thermal Engineering 61(2): 500-506. http://dx.doi.org/10.1016/j.applthermaleng.2013.07.043

Pourmahmoud, N.; Hasanzadeh, A.; Rafiee, S. E., et al. 2012. Three dimensional numerical investigation of effect of convergent nozzles on the energy separation in a vortex tube, Heat and Technology 30(2): 133-140.

http://dx.doi.org/10.18280/ijht.300219

Pourmahmoud, N.; Rafiee, S. E.; Rahimi, M., et al. 2013. Numerical energy separation analysis on the commercial Ranque-Hilsch vortex tube on basis of application of different gases, Scientia Iranica 20(5): 1528-1537.

Rafiee, S. E.; Rahimi, M. 2013. Experimental study and threedimensional (3D) computational fluid dynamics (CFD) analysis on the effect of the convergence ratio, pressure inlet and number of nozzle intake on vortex tube performance - validation and CFD optimization, Energy 63: 195-204. http://dx.doi.org/10.1016/j.energy.2013.09.060

Rafiee, S. E.; Rahimi, M.; Pourmahmoud, N. 2013. Three-dimensional numerical investigation on a commercial vortex tube based on an experimental model - Part I: optimization of the working tube radius, Heat and Technology 31(1): 49-56.

Rafiee, S. E.; Sadeghiazad, M. M. 2014a. Three-dimensional and experimental investigation on the effect of cone length of throttle valve on thermal performance of a vortex tube using k- $\varepsilon$ turbulence model, Applied Thermal Engineering 66(1-2): 65-74.

http://dx.doi.org/10.1016/j.applthermaleng.2014.01.073

Rafiee, S. E.; Sadeghiazad, M. M. 2014b. 3D CFD exergy analysis of the performance of a counter flow vortex tube, Heat and Technology 32 (1-2): 71-77.

Rafiee, S. E.; Ayenehpour, S.; Sadeghiazad, M. M. 2015a. A study on the optimization of the angle of curvature for a Ranque-Hilsch vortex tube, using both experimental and full Reynolds stress turbulence numerical modelling, Heat and Mass Transfer 33(1): 83-90.

Rafiee, S. E.; Sadeghiazad, M. M. 2015. 3D numerical analysis on the effect of rounding off edge radius on thermal separation inside a vortex tube, Heat and Technology 33(1): 83-90. http://dx.doi.org/10.18280/ijht.330112

Rafiee, S. E.; Sadeghiazad, M. M.; Mostafavinia, N. 2015b. Experimental and numerical investigation on effect of convergent angle and cold orifice diameter on thermal performance of convergent vortex tube, Thermal Science and Engineering Applications 7(4).

Ranque, G. J. 1933. Experiments on expansion in a vortex with simultaneous exhaust of hot air and cold air, Le Journal de Physique et le Radium 4: 112-114.

Shamsoddini, R.; Hossein Nezhad, A. 2010. Numerical analysis of the effects of nozzles number on the flow and power of cooling of a vortex tube, Refrigeration 33: 774-82. http://dx.doi.org/10.1016/j.ijrefrig.2009.12.029 
Skye, H. M.; Nellis, G. F.; Klein, S. A. 2006. Comparison of CFD analysis to empirical data in a commercial vortex tube, Refrigeration 29: 71-80.

http://dx.doi.org/10.1016/j.ijrefrig.2005.05.004

Suresh Kumar, G.; Padmanabhan G.; Dattatreya Sarma, B. 2014. Optimizing the temperature of hot outlet air of vortex tube using Taguchi Method, Procedia Engineering 97: 828-836. http://dx.doi.org/10.1016/j.proeng.2014.12.357

Thakare, H. R.; Parekh, A. D. 2015. Computational analysis of energy separation in counter - flow vortex tube, Energy 85: 62-77. http://dx.doi.org/10.1016/j.energy.2015.03.058

Valipour, M. S.; Niazi, N. 2011. Experimental modeling of a curved Ranque-Hilsch vortex tube refrigerator, Refrigeration 34: 1109-1116.

http://dx.doi.org/10.1016/j.ijrefrig.2011.02.013

Xue, Y.; Arjomandi, M.; Kelso, R. 2013a. Energy analysis within a vortex tube, Experimental Thermal and Fluid Science 52(January): 139-145.

Xue, Y.; Arjomandi, M.; Kelso, R. 2013b. Experimental study of the thermal separation in a vortex tube, Experimental Thermal and Fluid Science 46: 175-182. http://dx.doi.org/10.1016/j.expthermflusci.2012.12.009

Exair Corporation. Vortex tubes and spot cooling products [online], [cited 18 March 2015]. Available from Internet: http://www.exair.com 